\title{
Pathological Alteration of FoxO3a Activity Promotes Idiopathic Pulmonary Fibrosis Fibroblast Proliferation on Type I Collagen Matrix
}

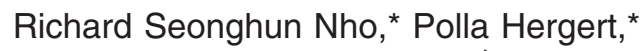 \\ Judith Kahm, ${ }^{*}$ Jose Jessurun, ${ }^{\dagger}$ and Craig Henke* \\ From the Departments of Medicine* and Lab Medicine and \\ Pathology, ${ }^{\dagger}$ University of Minnesota, Minneapolis, Minnesota
}

Idiopathic pulmonary fibrosis (IPF) is a prevalent, progressive, and incurable fibroproliferative lung disease. The phenotype of IPF fibroblasts is characterized by their ability to elude the proliferation-suppressive properties of polymerized type I collagen. The mechanism underlying this pathological response is incompletely understood but involves aberrant activation of the phosphatidylinositol 3-kinase-Akt signaling pathway owing to inappropriately low phosphatase and tensin homolog phosphatase activity. Akt can phosphorylate and inactivate the forkhead box $\mathrm{O} 3 \mathrm{a}$ (FoxO3a) transcriptional factor, which, when transcriptionally active, increases the expression of the CDK inhibitor p27 and promotes cell cycle arrest. Herein, we demonstrate that IPF fibroblasts display high levels of inactive FoxO3a compared with nonfibrotic control fibroblasts because of their high Akt activity. We found that p27 levels are decreased in IPF compared with control fibroblasts cultured on polymerized collagen. Furthermore, overexpression of FoxO3a in IPF fibroblasts increases p27 levels and suppresses the ability of IPF fibroblasts to proliferate on polymerized collagen. In contrast, the expression of dominant-negative FoxO3a augmented control fibroblast proliferation. IHC examination of fibroblastic foci in IPF lung tissue demonstrates the presence of inactive $\mathrm{FoxO} 3 \mathrm{a}$ in cells within fibroblastic foci. These data indicate that the ability of IPF fibroblasts to circumvent the proliferation-suppressive properties of polymerized collagen involves inactivation of FoxO3a by high Akt activity, resulting in downregulation of p27. (Am J Pathol 2011, 179:2420-2430; DOI: 10.1016/j.ajpath.2011.07.020)

Idiopathic pulmonary fibrosis (IPF) is a condition in which the lung parenchyma becomes progressively scarred over time. Consequently, this pathological condition, this distorted pulmonary architecture, severely disrupts lung function, often with fatal consequences. ${ }^{1,2}$ However, the pathogenesis of this deadly disease is not well understood and treatment to cure IPF is not available. During tissue repair, normal lung fibroblasts sense type I collagen matrix via integrins and the induction of key integrinmediated signaling pathways suppress fibroblast proliferation, thus limiting the fibroblast response. ${ }^{3-5}$ Our prior studies $^{6,7}$ demonstrate that, when normal lung fibroblasts attach to polymerized collagen via $\beta 1$ integrin, phosphatase and tensin homolog (PTEN) activity increases, inhibiting phosphatidylinositol 3-kinase (PI3K)-Akt function. In contrast, IPF fibroblasts have low PTEN activity, rendering inappropriately high PI3K-Akt activity on collagen. ${ }^{8,9}$ Thus, the IPF fibroblast phenotype is characterized by an aberrant integrin-mediated signaling pathway, enabling these cells to elude the proliferation-suppressing properties of polymerized collagen.

Forkhead box $\mathrm{O} 3 \mathrm{a}$ (FoxO3a) is a transcription factor that activates the CDK inhibitor protein p27. Up-regulation of FoxO3a activity increases p27 expression, which promotes cell cycle inhibition. ${ }^{10-12}$ Seminal studies ${ }^{13-15}$ have shown that FoxO3a activity is abnormally suppressed in several cancers. These observations link FoxO3a function to human disease. Several upstream proteins regulate $\mathrm{FoxO} 3 \mathrm{a}$ activity by phosphorylating many of its Ser-Thr residues. ${ }^{16-18}$ Among them, Akt is an important protein kinase in regulating its activity by phosphorylating a crucial Ser253 residue. ${ }^{19,20}$ Phosphorylation of FoxO3a by Akt retains FoxO3a in the cytoplasm, thereby inhibiting its activity. ${ }^{21-23}$ We found that $\beta 1$ integ-

Supported by grants from the NIH (R21 RHL096567A to R.S.N. and C.H. and P01 HL 091775 to C.H.), a Pulmonary Fibrosis Research grant from the American Lung Association (to R.S.N.), and a Scientist Development grant from the American Heart Association (to R.S.N.).

Accepted for publication July 29, 2011

Supplemental material for this article can be found at $h$ ttp://ajp. amjpathol.org or at doi: 10.1016/j.ajpath.2011.07.020.

Address reprint requests to Richard Seonghun Nho, Ph.D., MMC 276, Department of Medicine, 420 Delaware St. SE, Minneapolis, MN 55455. E-mail: nhoxx002@umn.edu. 
rin-collagen interaction suppresses FoxO3a function in a PTEN-PI3K-Akt-dependent fashion. ${ }^{18}$ Herein, we demonstrate that, in IPF fibroblasts, the FoxO3a transcription factor, which inhibits cell cycle progression by up-regulating the cell cycle inhibitor p27, is largely inactive. We show that inappropriately high Akt activity owing to low PTEN function phosphorylates and inactivates FoxO3a in IPF fibroblasts. We found that, in IPF fibroblasts, inactive FoxO3a is associated with low p27 levels. More important, we demonstrate that this inappropriately high Akt activity inactivates $\mathrm{FoxO} 3$ a and confers IPF fibroblasts with the ability to circumvent the proliferation-suppressive effects of polymerized collagen.

\section{Materials and Methods}

\section{Cell Culture and Type I Collagen Matrices}

Eight control and seven IPF primary fibroblast lines were established and analyzed for this study. Cell lines were derived from lungs removed at transplantation or death. The diagnosis of IPF was supported by medical history, physical examination findings, pulmonary function test results, and typical high-resolution chest computed tomography findings of IPF. In all cases, the diagnosis of IPF was confirmed by microscopic analysis of lung tissue and demonstrated the characteristic morphological findings of usual interstitial pneumonia. All patients fulfilled the criteria for the diagnosis of IPF as established by the American Thoracic Society and the European Respiratory Society. Eight control primary adult human lung fibroblast lines were established from histologically normal lung tissue adjacent to carcinoid tumor or adjacent to radiation-induced fibrotic lung tissue. Primary lung fibroblast lines were generated by explant culture and cultured in high-glucose Dulbecco's modified Eagle's medium (DMEM) containing 10\% fetal calf serum. Fibroblasts were used between passages five and seven. Cells were characterized as fibroblasts as previously described. ${ }^{8}$ Use of human tissues was approved by the Institutional Review Board at the University of Minnesota, Minneapolis. FoxO3a $^{-1-}$ mouse embryonic fibroblasts were obtained from Dr. Noboru Motoyama (National Center for Geriatrics and Gerontology, Obu, Aichi, Japan). PTEN ${ }^{-1-}$ and PTEN $^{+/+}$mouse fibroblasts were provided by Deane F. Mosher (University of Wisconsin-Madison). These cells were maintained in DMEM plus $10 \%$ fetal bovine serum, $1 \%$ penicillin, and $1 \%$ streptomycin. Type I collagen solution (Vitrogen 1000) was obtained from Cohesion (Palo Alto, CA).

\section{Collagen Matrices}

Three-dimensional polymerized collagen matrices (final concentration, $2 \mathrm{mg} / \mathrm{mL}$ ) were prepared by neutralizing the collagen solution with a one-sixth volume of six times DMEM medium, diluting to a final volume with one times DMEM, and incubating the solution at $37^{\circ} \mathrm{C}$ for 3 to 4 hours before use. ${ }^{18}$

\section{Antibodies and Chemicals}

For Western blot analysis, anti-PTEN antibody was obtained from Santa Cruz Biotechnology (Santa Cruz, CA). Anti-Akt antibody was obtained from Cell Signaling Technologies (Danvers, MA). FoxO3a and phosphorylated FoxO3a ( $p-F o x O 3 a ;$ Ser253) antibodies were obtained from Millipore (Billerica, MA) and Cell Signaling Technologies. The p27 antibody was obtained from Chemicon International (Temecula, CA). For immunohistochemistry $(\mathrm{IHC})$ and immunofluorescence, $\mathrm{p}-\mathrm{FoxO} \mathrm{O} a$ antibody was obtained from Santa Cruz Biotechnology. $\alpha$-Smooth muscle actin antibody was obtained from Vector Laboratories (Burlingame, CA). $\beta_{1}$ Integrin blocking antibody P5D2 was also produced from hybridoma culture. $\beta_{1}$ Integrinactivating monoclonal antibody TS2/16 was produced from hybridoma culture (American Type Culture Collection, HB-243). Akt inhibitor was obtained from Calbiochem (San Diego, CA).

\section{Adenovirus and FoxO3a Constructs}

For PTEN adenovirus construction, wild-type (WT) PTEN CDNA was generated from normal human lung fibroblasts (HLF-210) by RT-PCR. The forward and reverse primers used for WT PTEN (PW), which span the entire coding region of PTEN, are 5'-CTACTCGAGGCTCCCAGACATGAC-3' and 5'-ACGCTCGAGATAAAAAAAAATTCAG-3', respectively. The lipid and protein phosphatase truncated mutant was generated by PCR with forward and reverse primers 5'-CAGCTCGAGGGACGAACTGGTGTAA-3' and 5'-ACGCTCGAGATAAAAAAAAAT TCAG-3', respectively. The amplified products were cloned into MIGR1-IRES-green fluorescent protein (GFP), followed by Sall and EcoRI digestion. The PW MIGR1-IRES-GFP fragments were then cloned into the adenoviral vector, pAxCAwt (obtained from Dakara Bio. Inc., Otsu, Shiga, Japan), and adenovirus titer was measured by the plaque method in $0.5 \%$ soft agar. The cells were infected with adenoviral vectors at a multiplicity of infection of 1:20. Adenovirus-expressing HA-tagged Akt with a c-Src myristolyation sequence fused in frame to the $\mathrm{N}$-terminus [hyperactive Akt (HA)], HA-tagged Akt dominant-negative mutant (T308A and S473A), and WT FoxO3a were obtained from Vector Biolabs, Eagleville, PA. Adenovirusexpressing GFP-tagged WT FoxO3a, dominant-negative FoxO3a (DF) with the deletion of the transactivation domain from the C-terminus, and empty vector were obtained from Vector Biolabs. Control or IPF fibroblasts were infected with adenovirus expressing PTEN, Akt, or empty vector in $2 \mathrm{~mL}$ of tissue culture dishes. A total of $1 \times 10^{5}$ to $2 \times 10^{5}$ control or IPF fibroblasts per milliliter of DMEM were infected with $1 \times 10^{6}$ plaque-forming units of each adenovirus for assay.

\section{Isolation of Cytoplasmic and Nuclear Fractions}

For the separation of cytoplasmic and nuclear fractions, NE-PER Nuclear and Cytoplasmic extraction reagents from Thermo Scientific (Pittsburgh, PA), were used. Briefly, control and IPF fibroblasts were collected and 
suspended in $100 \mu \mathrm{L}$ of cytoplasmic extraction reagent I solution (Thermo Scientific). Tube-containing cells were then vortex mixed, and $5.5 \mu \mathrm{L}$ of cytoplasmic extraction reagent II was added, followed by incubation in ice for 5 minutes. Cytoplasmic extract was then collected by centrifugation at maximum speed for 5 minutes. The insoluble nuclear fraction was resuspended in $50 \mu \mathrm{L}$ of nuclear extraction reagent buffer and vortex mixed for $15 \mathrm{sec}-$ onds every 10 minutes. The nuclear fraction was finally collected by centrifugation at maximum speed $(16,000 \times$ g) for 10 minutes for analysis.

\section{IHC and Immunofluorescence}

Human lung tissue was prepared as previously described. ${ }^{8,9}$ After embedding in paraffin, sections $(5-\mu \mathrm{m}$ thick) were cut and mounted onto polylysine-coated slides. The paraffin sections were deparaffinized in xylene, rehydrated through a graded series of methanol, and placed in a $95^{\circ} \mathrm{C}$ to $100^{\circ} \mathrm{C}$ water bath for 30 minutes in citrate buffer ( $\mathrm{pH}$ 6.0) for antigen retrieval. The sections were quenched with $3 \%$ hydrogen peroxide in PBS and incubated in normal goat serum for 30 minutes to block nonspecific binding of secondary antibodies. Endogenous avidin and biotin binding sites were blocked by sequential incubation for 15 minutes. The sections were then incubated overnight at $4^{\circ} \mathrm{C}$ with $\mathrm{p}$-FoxO3a or FoxO3a in PBS (polyclonal antibody, 1:50; Santa Cruz Biotechnology). IHC was performed by the avidin-biotin peroxidase complex method. The Vectastain Elite ABC kit was used for all experiments, as instructed by the manufacturer (Vector Laboratories). Immunoreactivity was detected with 3,3'-diaminobenzidine as a peroxidase substrate, and the sections were counterstained with hematoxylin. The Leica Leitz DMRB microscope (Leica, Wetzlar, Germany) was used to acquire all images. PhotoShop CS5 (Adobe, San Jose, CA) was used for image processing. For the immunofluorescence assay, IPF fibroblasts cultured on polymerized collagen were fixed for 10 minutes in cold methanol, rinsed with PBS, and blocked in 5\% donkey serum in PBS buffer for 30 minutes at room temperature. Cells were then incubated with p-FoxO3a primary antibody overnight at $4^{\circ} \mathrm{C}$, washed three times with PBS, and incubated in donkey anti-rabbit Cy 2 (Jackson Immunoresearch, West Grove, PA) secondary antibody diluted in PBS for 45 minutes at room temperature in the dark. After rinsing with PBS, the slides were incubated in DAPI in PBS at room temperature for 20 minutes in the dark. After washing three times, ProLong Gold antifade reagent (Life Technologies, Eugene, OR) was added to the coverslip and the slides were sealed with nail polish. Specimens were imaged with the Zeiss Axiovert 200 microscope (Zeiss, Gottigen, Germany).

\section{Cell Proliferation Assay}

The number of viable proliferating cells was determined using the CellTiter 96 Aqueous One Solution Cell Proliferation Assay [3-(4,5-dimethylthiazol-2-yl)-5-(3-carboxymethoxyphenyl)-2-(4-sulfophenyl)-2H-tetrazolium (MTS) assay; Promega (Madison, WI)]. Briefly, normal human lung or IPF fibroblasts were infected with WT, DF, HA, or dominant-negative Akt (DA) for 24 hours. A total of $2 \times$ $10^{3}$ human lung fibroblasts were then grown in $100 \mu \mathrm{L}$ of DMEM on 96-well plates overnight. CellTiter 96 Aqueous One Solution Reagent, $20 \mu \mathrm{L}$, was added to each well of the 96-well plate, followed by incubation for 2 hours at $37^{\circ} \mathrm{C}$ in a humidified $5 \% \mathrm{CO}_{2}$ atmosphere. The absorbance at $490 \mathrm{~nm}$, using a 96-well plate reader, was measured. For the cell counting assay, cell numbers were counted using the Coulter counter (Beckman Coulter, Miami, FL). Attached cells were then trypsinized, collected, and counted.

\section{Statistical Analysis}

Data are expressed as the mean \pm SD. Experiments were performed three times. Paired evaluations were made for experimental and control conditions within each experiment, and significance was determined by the Student's $t$-test. The significance level was set at $P<0.05$.

\section{Results}

\section{Inappropriately High Akt Activity Phosphorylates and Inactivates FoxO3a in IPF Fibroblasts}

We have previously shown that when normal fibroblasts attach to polymerized collagen, high PTEN activity inhibits their proliferation. ${ }^{8}$ In contrast, IPF fibroblasts display inappropriately high proliferation on polymerized collagen because of aberrantly high Akt activity, resulting from low membrane PTEN expression. Because Akt suppresses FoxO3a by phosphorylating the Ser253 residue, we hypothesized that FoxO3a activity is abnormally low in IPF fibroblasts on polymerized collagen, thereby promoting fibroblast proliferation. To examine this hypothesis, we first measured the level of phosphorylated (inactive) FoxO3 in control $(n=5)$ and IPF $(n=5)$ fibroblasts on polymerized collagen. The level of p-FoxO3a has been used as a surrogate marker of FoxO3a activity. The p-FoxO3a levels were high in IPF compared with control fibroblasts when cultured on polymerized collagen (Figure 1A). p-FoxO3a-actin expression ( $n=8$ control fibroblasts, and $n=7$ IPF fibroblasts) was overall more than threefold higher in IPF compared with control fibroblasts (Figure 1B). These data indicate that FoxO3a activity is low in response to IPF fibroblast attachment to collagen. Furthermore, the overall level of FoxO3a expression was high in control compared with IPF fibroblasts on collagen matrix (Figure 1, C and D). Our data demonstrate that, in response to IPF fibroblast attachment to collagen, FoxO3a activity is aberrantly low.

Because the level of p-FoxO3a is high in IPF fibroblasts, we also sought to address whether high Akt activity is responsible for phosphorylation and inactivation of FoxO3a in IPF fibroblasts. We first measured the level of FoxO3a phosphorylation in the presence or absence of an Akt inhibitor. For this assay, IPF fibroblasts were preincubated with various concentrations of an Akt inhibitor 
A
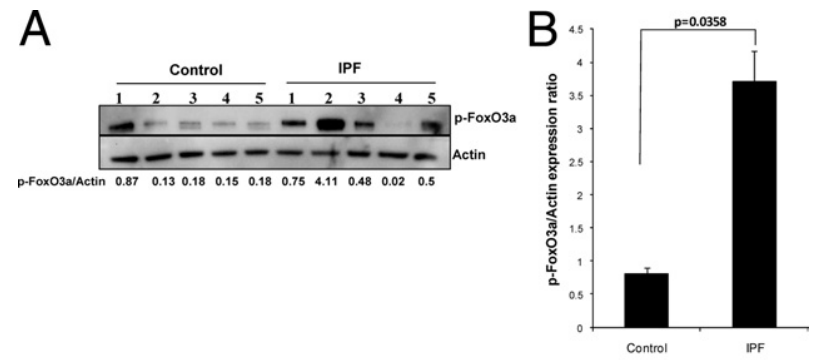

C

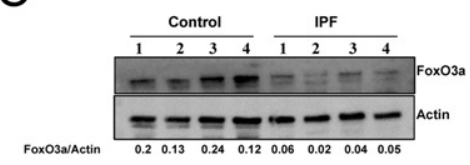

D

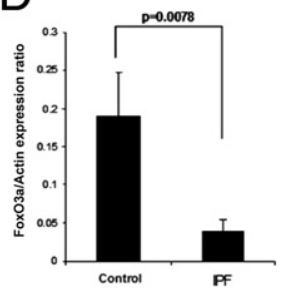

Figure 1. Phosphorylated (inactive) FoxO3a expression is high in IPF fibroblasts. Serum-starved control and IPF fibroblasts were plated on polymerized collagen for 24 hours, and Western blot analysis was performed with antibodies, as described. A: Ser253-p-FoxO3a protein levels were measured. Actin was used as a loading control. The p-FoxO3a/actin expression ratio was measured using densitometry. A representative Western blot analysis for p-FoxO3a levels in control and IPF fibroblasts cultured on polymerized collagen is shown ( $n=5$ control fibroblasts and $n=5$ IPF fibroblasts). B: p-FoxO3a/actin expression in control and IPF fibroblasts on polymerized collagen was measured ( $n=8$ control fibroblasts, and $n=7$ IPF fibroblasts; $P<0.04$ versus control). C: FoxO3a protein levels in control $(n=4)$ and IPF $(n=4)$ fibroblasts were measured. A representative Western blot analysis for FoxO3a expression levels in control or IPF fibroblasts is shown. Actin was used as a loading control. The FoxO3a/actin ratio was measured by densitometry. D: FoxO3a/actin expression on polymerized collagen gels was measured by densitometry in control $(n=5)$ and IPF $(n=4)$ fibroblasts. $P<0.01$ versus control.

or dimethylsulfoxide control and plated on type I collagen, and the level of p-FoxO3a was measured. p-FoxO3a levels were progressively diminished in a dose-dependent fashion (Figure 2A). We also examined FoxO3a levels using an antibody that recognizes FoxO3a expression in general (not specific for p-FoxO3a). Unlike p-FoxO3a expression, we found that FoxO3a levels were maintained high when a high dose of Akt inhibitor was used (Figure 2A). These data suggest that during IPF fibroblast interaction with collagen, Akt phosphorylates and suppresses FoxO3a activity.

\section{PTEN-Akt Axis Regulates FoxO3a Activity in IPF Fibroblasts}

Because IPF fibroblasts display inappropriately low PTEN activity, resulting in high Akt activity, we next examined the role of PTEN in regulating FoxO3a via Akt. To begin to address this issue, we first used PTEN ${ }^{-1-}$ cells to examine FoxO3a activity. PTEN ${ }^{-1-}$ cells were reconstituted with PW or mutant PTEN (PD) protein, and the levels of FoxO3a and p-FoxO3a were assessed. Overexpression of PW completely suppressed the expression of $\mathrm{p}-\mathrm{FoxO} 3 \mathrm{a}$, despite an increase in the overall level of FoxO3a (Figure 2B). In contrast, mutant PTEN failed to inhibit FoxO3a activity. To verify this finding, control lung fibroblasts were first infected with an adenoviral vector expressing PW, phosphatase-dead mutant PTEN, or

empty vector (GFP), and the level of p-FoxO3a was measured. The level of $\mathrm{p}$-FoxO3a was barely detectable when $\mathrm{PW}$ was overexpressed in control fibroblasts (Figure 2C). In contrast, the overall level of FoxO3a remained high when PW was overexpressed. However, when mutant PTEN was overexpressed, the level of p-FoxO3a was increased compared with control, whereas the FoxO3a level was markedly diminished (lane 2). Interestingly, the FoxO3a/p-FoxO3a expression ratio was not significantly altered when PW was expressed in control fibroblasts (Figure 2C), suggesting that further induction of PTEN protein does not significantly increase FoxO3a activity, presumably because of the presence of high baseline PTEN activity when these cells interact with polymerized collagen.

We next analyzed the level of FoxO3a phosphorylation in control fibroblasts using HA or DA constructs to further examine the effect of Akt on FoxO3a function. HA increased p-FoxO3a levels compared with control (Figure 2D). Interestingly, the level of $\mathrm{p}$-FoxO3a remained suppressed in control fibroblasts. This suggests that DA cannot further suppress the already low level of Akt activity resulting from high PTEN activity in control fibroblasts on collagen (Figure 2D). We next examined the role of the PTEN-Akt axis in regulating $\mathrm{p}$-FoxO3a levels in IPF fibroblasts interacting with polymerized collagen. The overexpression of PW in IPF fibroblasts decreased the level of p-FoxO3a, whereas mutant PTEN had a minimal effect on the already high level of p-FoxO3a in IPF fibroblasts compared with the GFP control (Figure 2E). Furthermore, the analysis of the FoxO3a/p-FoxO3a expression ratio showed that, compared with control, HA did not significantly increase the level of p-FoxO3a (Figure 2F). In contrast, the FoxO3a/p-FoxO3a expression ratio was high when DA was overexpressed in IPF fibroblasts on collagen matrix (lane 2). Taken together, these data demonstrate that the PTEN-Akt axis regulates FoxO3a function in response to fibroblast attachment to polymerized collagen. Our data indicate that when IPF fibroblasts interact with polymerized collagen, low PTEN function inappropriately activates Akt, thereby phosphorylating and inactivating FoxO3a.

\section{Nuclear FoxO3a Levels Are Low in IPF Fibroblasts Cultured on Polymerized Collagen}

On activation, the FoxO3a transcription factor translocates from the cytoplasm into the nucleus. In contrast, FoxO3a that has been phosphorylated by Akt is located predominantly in the cytoplasm and is inactive. ${ }^{20,21} \mathrm{Be}$ cause IPF fibroblasts display low FoxO3a activity, we hypothesized that FoxO3a will be largely located in the cytoplasm when IPF fibroblasts interact with polymerized collagen. To examine this hypothesis, we isolated and measured the nuclear and cytoplasmic fractions of FoxO3a and p-FoxO3a in control and IPF fibroblasts cultured on polymerized collagen. Consistent with our finding that FoxO3a is largely inactive in IPF fibroblasts, we found that FoxO3a was barely detectable in the nucleus of IPF fibroblasts (Figure 3A), whereas FoxO3a expres- 
A

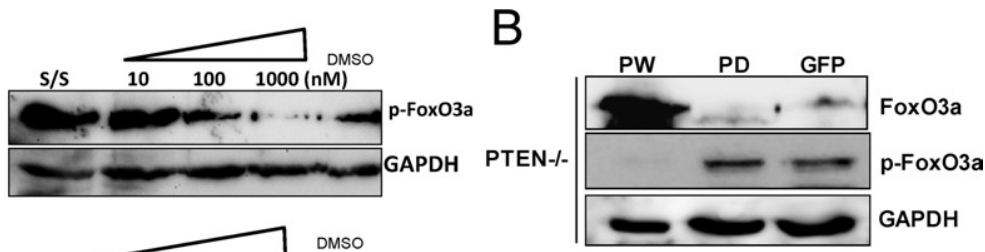

C
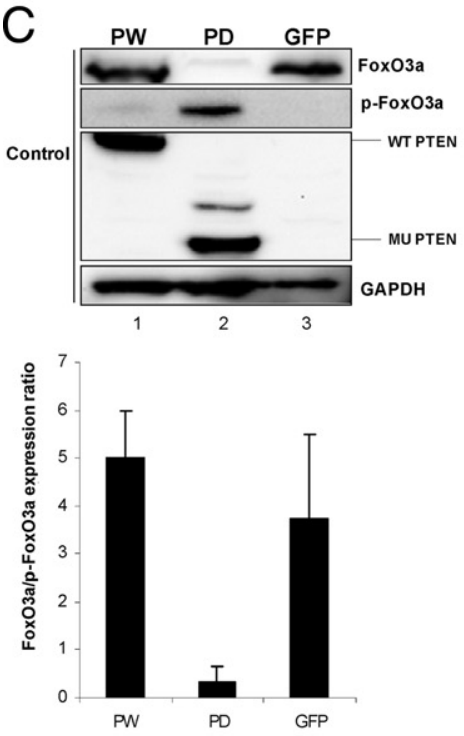

E
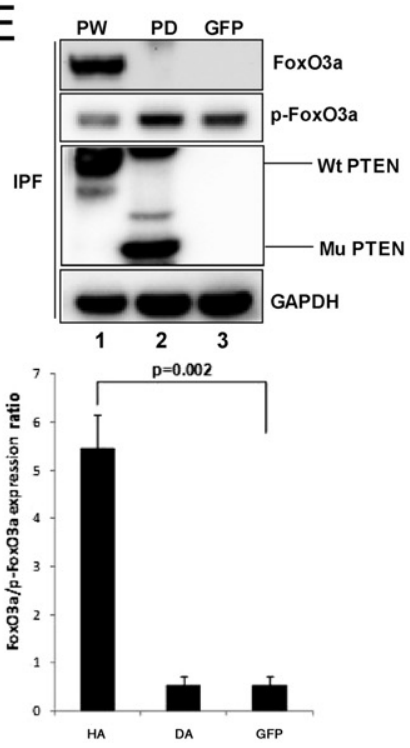

D
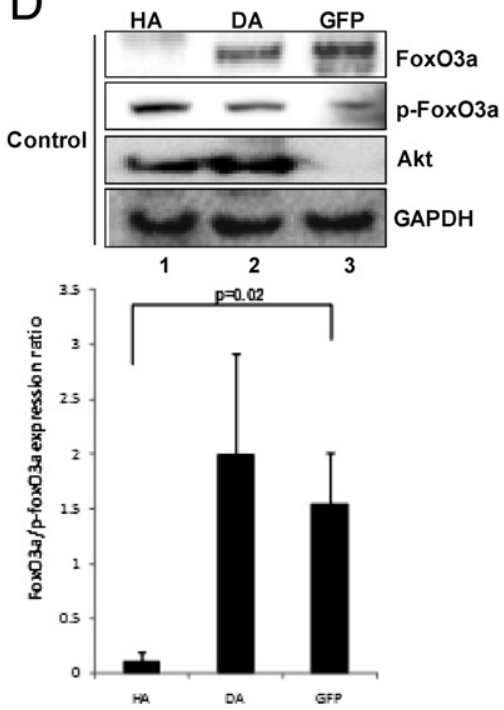

$\mathrm{F}$
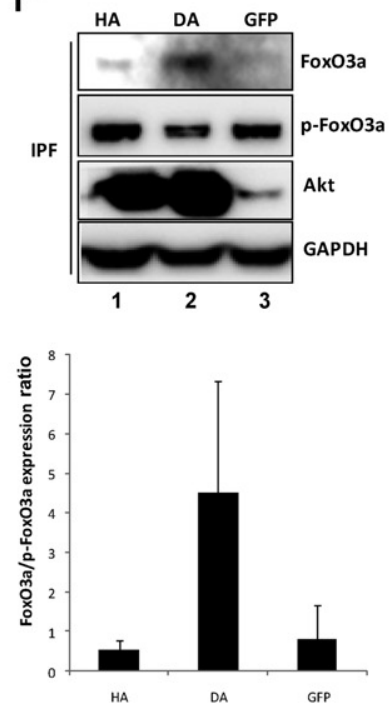

Figure 2. Inhibition of Akt decreases p-FoxO3a levels in IPF fibroblasts. Serum-starved IPF fibroblasts were pre-incubated with an Akt inhibitor, ranging from 10 to 1000 $\mathrm{nmol} / \mathrm{L}$, and plated on collagen matrix for 60 minutes. A: p-FoxO3a (top) and FoxO3a (bottom) levels were examined by Western blot analysis. Glyceraldehyde-3-phosphate dehydrogenase (GAPDH) was used as a loading control Dimethylsulfoxide (DMSO)-treated cells were used as a control. B: PTEN ${ }^{-1-}$ cells were infected with adenoviral constructs expressing wild-type PTEN (PW), phosphatasedeleted PTEN (PD), or empty vector (GFP), and cells were cultured on polymerized collagen for 24 hours. Western blot analysis was performed to measure FoxO3a, p-FoxO3a, and GAPDH protein levels. C: Top: Control fibroblasts infected with PW, PD, or empty vector GFP were cultured on polymerized collagen for 24 hours, and FoxO3a, p-FoxO3, and PTEN levels were measured. GAPDH was used as a loading control. Bottom: The FoxO3a/p-FoxO3a expression ratio in control fibroblasts infected with PW, PD, or empty vector (GFP) was measured by densitometry. D: Top: Control human lung fibroblasts infected with an adenovirus expressing HA, DA, or empty vector (GFP) were cultured on polymerized collagen for 24 hours, and Western blot analysis was performed to measure FoxO3a, p-FoxO3a, Akt, and GAPDH levels. Bottom: The FoxO3a/p-FoxO3a expression ratio was quantified by densitometry. E: Top: IPF fibroblasts overexpressing PW, PD, or empty vector (GFP) were cultured on polymerized collagen for 24 hours, and FoxO3a, p-FoxO3a, and PTEN levels were measured. GAPDH was used as a loading control. Bottom: The FoxO3a/p-FoxO3a expression ratio was quantified by densitometry. F: Top: IPF fibroblasts infected with an adenovirus expressing HA, DA, or empty vector (GFP) were cultured on polymerized collagen for 24 hours, and Western blot analysis was performed to measure FoxO3a, p-FoxO3a, Akt, and GAPDH levels. Bottom: The FoxO3a/p-FoxO3a protein expression ratio was measured by densitometry. sion was high in the nuclear fraction of control fibroblasts (lane 3). The calculation of the FoxO3a/p-FoxO3a expression ratio using densitometry illustrates that nuclear FoxO3a is low in IPF fibroblasts compared with control (Figure 3B). In contrast, we found that the level of phosphorylated or inactive FoxO3a was elevated in the cytoplasm of IPF fibroblasts cultured on polymerized collagen compared with that of control fibroblasts (Figure $3 \mathrm{~A}$ ). The ratio of FoxO3a/p-FoxO3a in the cytoplasmic fraction of IPF fibroblasts was approximately 32\% lower compared with that of control fibroblasts (Figure 3B). To verify our Western blot analysis data, we further examined the location of $\mathrm{p}-\mathrm{FoxO} 3 \mathrm{a}$ in IPF fibroblasts on collagen using immunofluorescence. We found that $\mathrm{p}$-FoxO3a is mainly located in the cytoplasm but that some p-FoxO3a can also be found in the nucleus (see Supplemental Figure 


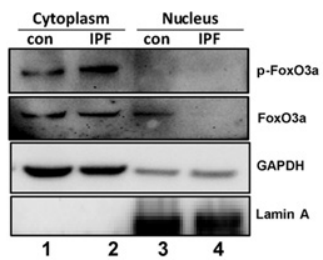

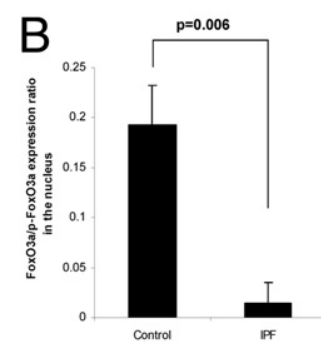
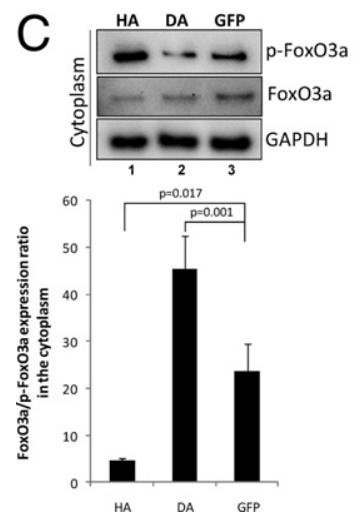

Figure 3. Nuclear FoxO3a levels are low in IPF fibroblasts. A: Serum-starved control (con) and IPF fibroblasts were cultured on polymerized collagen for 24 hours. Cytoplasmic and nuclear fractions were collected, and p-FoxO3a and FoxO3a levels were measured by Western blot analysis. Glyceraldehyde3-phosphate dehydrogenase (GAPDH) was used as a cytoplasmic loading control. Lamin A was used as a nuclear loading control. B: The FoxO3a/pFoxO3a expression ratio in the nucleus (left) or cytoplasm (right) was quantified in control and IPF fibroblasts cultured on polymerized collagen by densitometry. C: Top: IPF fibroblasts infected with an adenovirus expressing HA, DA, or empty vector (GFP) were cultured on polymerized collagen for 24 hours, and the cytoplasmic fraction was separated. Western blot analysis was performed to measure p-FoxO3a and FoxO3a protein levels. GAPDH was used as a loading control for the cytoplasmic fraction. Bottom: The FoxO3a/p-FoxO3a expression ratio was quantified by densitometry. D: Top: IPF fibroblasts infected with an adenovirus expressing PW, PD, HA, DA, or empty vector (GFP) were cultured on polymerized collagen for 24 hours, and the nuclear fraction was separated. Western blot analysis was then performed to measure FoxO3a protein levels. Lamin A was used as a nuclear loading control. Bottom: Densitometric analysis of the FoxO3a/p-FoxO3a expression ratio in the nucleus.

S1A at http://ajp.amjpathol.org). Our results indicate that when IPF fibroblasts interact with polymerized collagen, FoxO3a is predominantly inactivated and largely located in the cytoplasm, whereas there is little active FoxO3a in the nucleus.

We next examined the role of Akt in regulating the cellular distribution of FoxO3a in IPF fibroblasts cultured on collagen. For this experiment, we assessed the level of $\mathrm{p}$-FoxO3a in the cytoplasm in IPF fibroblasts infected with an adenovirus expressing DA and HA. Inhibition of Akt by $\mathrm{DA}$ reduced the level of $\mathrm{p}-\mathrm{FoxO} 3 \mathrm{a}$ in the cytoplasm compared with GFP control (Figure 3C). In contrast, HA increased the level of phosphorylated (inactive) FoxO3a. The levels of $\mathrm{FoxO} 3 \mathrm{a}$ in the cytoplasm were relatively unaltered when HA or DA was expressed (Figure $3 \mathrm{C}$ ). The FoxO3a/p-FoxO3a expression ratio demonstrated that HA increased cytoplasmic p-FoxO3a, whereas DA modestly decreased the p-FoxO3a level in the cytoplasm (Figure 3C). We also examined whether FoxO3a levels were restored in the nucleus when Akt activity was inhibited. To test this, we overexpressed WT, mutant PTEN, $\mathrm{HA}$, and DA and measured the FoxO3a level in the nucleus of IPF fibroblasts on collagen. When PW was overexpressed, the level of nuclear FoxO3a increased compared with GFP control (Figure 3D). Consistent with this result, when DA was overexpressed, the level of FoxO3a in the nucleus was also significantly elevated (Figure 3D). These data strongly suggest that inappropriately high Akt activity in IPF fibroblasts promotes the accumulation of $\mathrm{p}-\mathrm{FoxO} 3 \mathrm{a}$ in the cytoplasm, where it is transcriptionally inactive.

\section{The Level of p27, a FoxO3a Target Protein, Is Low in IPF Fibroblasts}

The FoxO3a transcription factor up-regulates the expression of the CDK inhibitor protein p27, thus inhibiting cell proliferation. In contrast, p27 levels are suppressed in FoxO3a-null cells, facilitating proliferation. ${ }^{18}$ Because FoxO3a activity is low in IPF fibroblasts interacting with polymerized collagen, we next sought to analyze p27 expression in control and IPF fibroblasts cultured on collagen matrices as a function of time. We found that p27 expression was largely absent in IPF fibroblasts, whereas p27 expression increased in control fibroblasts cultured on polymerized collagen as a function of time (Figure 4A). To verify that FoxO3a regulates p27 expression in IPF fibroblasts, we overexpressed WT FoxO3a, DF, or empty vector in IPF fibroblasts. The level of p27 increased when WT FoxO3a was overexpressed in IPF fibroblasts, whereas p27 expression levels were not detectable in IPF fibroblasts infected with virus expressing DF or empty vector (Figure 4B). Similar results were also seen with the FoxO3a ${ }^{-1-}$ cells, in which, in response to reconstitution of FoxO3a, p27 expression was restored (Figure 4C). Because p27 expression was high when control fibroblasts were cultured on collagen, we also examined the effect of DF on p27 levels in control fibroblasts. The p27 expression was suppressed by the DF construct (Figure 4C). These data indicate that low FoxO3a activity in IPF fibroblasts results in decreased protein levels of p27.

Because high Akt activity inactivates FoxO3a in IPF fibroblasts, we next examined the effect of down-regulation of Akt on p27 expression. The overexpression of DA increased p27 levels in IPF fibroblasts cultured on polymerized collagen (Figure 4D). In contrast, p27 expression remained low when HA or empty vector was expressed in IPF fibroblasts (lanes 1 and 3). In a prior study, ${ }^{18}$ we found that $\beta 1$ integrin activation regulates FoxO3a expression. Therefore, we next examined whether $\beta 1$ integrin regulates p27 expression. To test this, we ligated control fibroblasts in suspension culture with the $\beta 1$ integrin activating antibody TS2/16, and p27 expression was measured. We found that p27 expression 

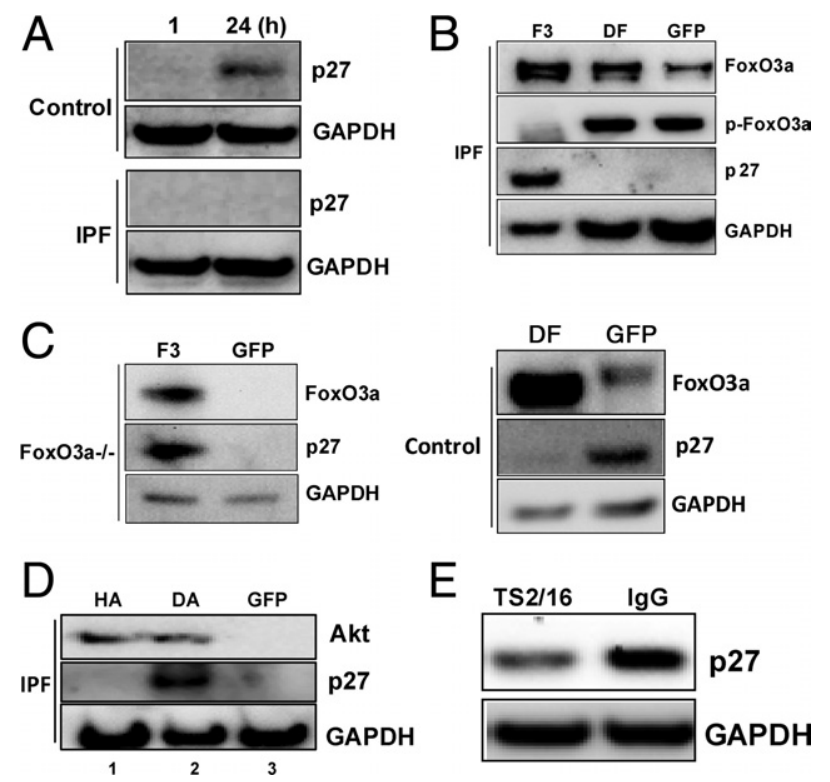

Figure 4. The p27 expression is low in IPF fibroblasts. A: Serum-starved control and IPF fibroblasts were cultured on polymerized collagen gels as a function of time, and p27 expression was measured by Western blot analysis. Glyceraldehyde-3-phosphate dehydrogenase (GAPDH) was used as a loading control. B: IPF fibroblasts were infected with an adenovirus expressing WT FoxO3a (F3), DF, or empty vector (GFP), and FoxO3a, p-FoxO3a, p27, and GAPDH expression levels were measured at 24 hours. C: Top: FoxO3a $a^{-1-}$ cells were reconstituted with F3 or empty vector (GFP), and FoxO3a, p27, and GAPDH levels were measured at 24 hours. Bottom: DF was expressed in control fibroblasts, and FoxO3a and p27 expression levels were measured at 24 hours. GAPDH was used as a loading control. D: HA, DA, or empty vector (GFP) was overexpressed in IPF fibroblasts, and p27 expression was measured at 24 hours. GAPDH was used as a loading control. E: Serum-starved control fibroblasts in suspension culture were ligated with a $\beta 1$ integrin-activating antibody (TS2/16) or an isotype control IgG antibody $(3 \mu \mathrm{g} / \mathrm{mL})$ for 1 hour. Cells were then collected and Western blot analysis was performed to measure p27 levels. GAPDH was used as a loading control.

was significantly low when cells were stimulated with TS2/16 (Figure 4E). In contrast, p27 expression remained high when fibroblasts were incubated with IgG isotype control. Taken together, our data showed that $\beta 1$ integrin regulates p27 expression.

\section{Overexpression of FoxO3a Increases p27 Expression Levels and Suppresses IPF Fibroblast Proliferation on Polymerized Collagen}

We previously found that polymerized collagen suppresses normal fibroblast proliferation, whereas IPF fibroblasts largely elude the proliferation-suppressive properties of polymerized collagen., ${ }^{8,9}$ Because p27 expression is low in IPF fibroblasts as the result of inactivation of FoxO3a by high Akt activity, this suggests that inactivation of FoxO3a and low p27 expression may underlie the ability of IPF fibroblasts to proliferate on polymerized collagen. To examine the role of $\mathrm{FoxO} 3 \mathrm{a}$ in regulating IPF fibroblast proliferation on polymerized collagen, we overexpressed WT FoxO3a or DF in IPF fibroblasts and assessed proliferation by the MTS assay. The overexpression of WT FoxO3a in IPF fibroblasts augmented the p27 level (Figure 5A) and suppressed IPF proliferation by $26 \%$ compared with control (Figure 5, B and C). In con-
A

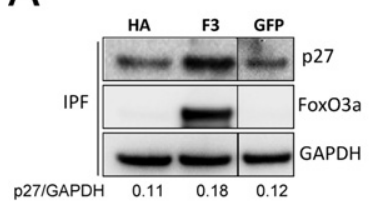

B
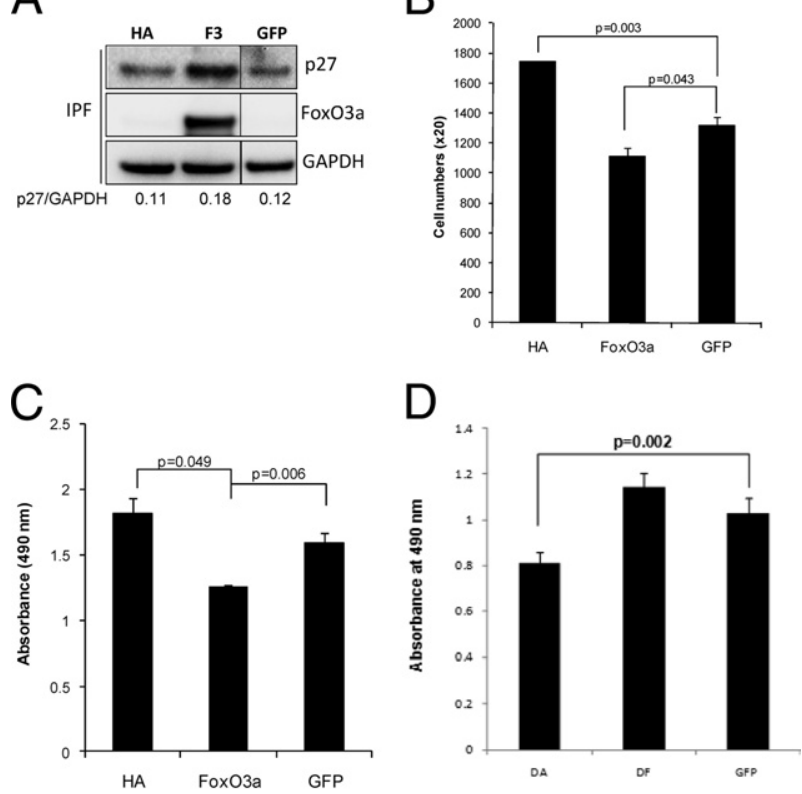

E

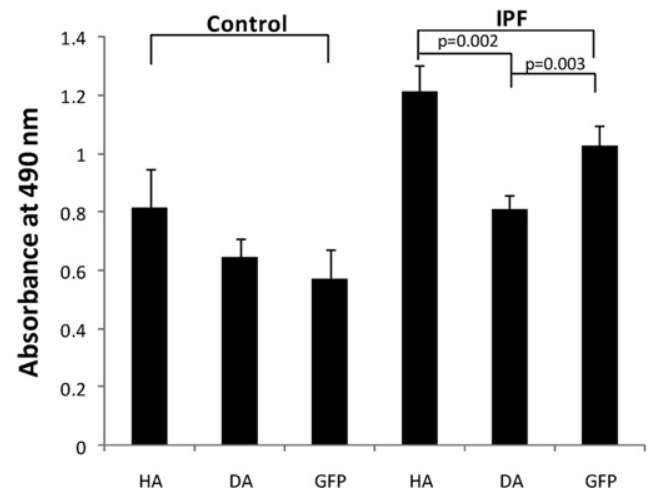

Figure 5. The overexpression of FoxO3a in IPF fibroblasts increases the p27 level and inhibits proliferation. A: IPF fibroblasts infected with adenovirus constructs expressing HA, WT FoxO3a (F3), or empty vector (GFP) were cultured on polymerized collagen for 72 hours in DMEM-containing serum. p27, FoxO3a, and glyceraldehyde-3-phosphate dehydrogenase (GAPDH) expression levels were measured by Western blot analysis. The p27/GAPDH expression ratio was quantified by densitometry. Western blot analysis images of GFP-expressed cells were from the same gel. B: A total of 20,000 IPF cells infected with adenovirus constructs expressing HA, FoxO3a, or empty vector (GFP) were cultured on polymerized collagen for 72 hours in the presence of serum, and cell numbers were counted as described in Materials and Methods. C: The proliferation of IPF fibroblasts infected with HA, FoxO3a, or GFP was quantified by MTS assay, as described in Materials and Methods. D: IPF fibroblasts were infected with an adenovirus expressing DA, DF, or GFP, and an MTS assay was performed as described. IPF fibroblast proliferation was significantly suppressed when DA was expressed, whereas the FoxO3a mutant had a minimal effect on IPF fibroblast proliferation. E: Control (left) or IPF (right) fibroblasts infected with adenovirus expressing HA, DA, or empty vector (GFP) were cultured on a 96-well plate, and an MTS assay was performed to measure proliferation at 24 hours, as previously described.

trast, the overexpression of DF had no significant effect on IPF fibroblast proliferation (Figure 5D). Interestingly, the overexpression of WT FoxO3a in control fibroblasts did not significantly alter proliferation, presumably because FoxO3a activity was already high in control fibroblasts cultured on polymerized collagen (data not shown). We next assessed the role of Akt in regulating control and IPF fibroblast proliferation on polymerized collagen. When HA was overexpressed, control fibroblast 

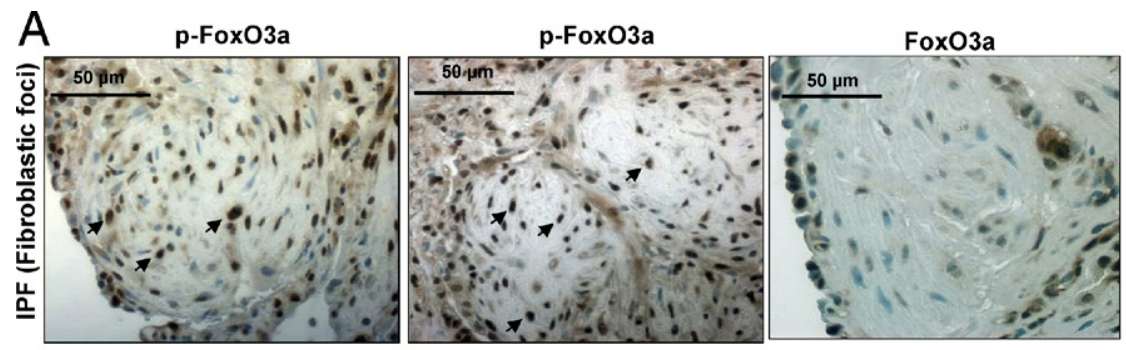

B
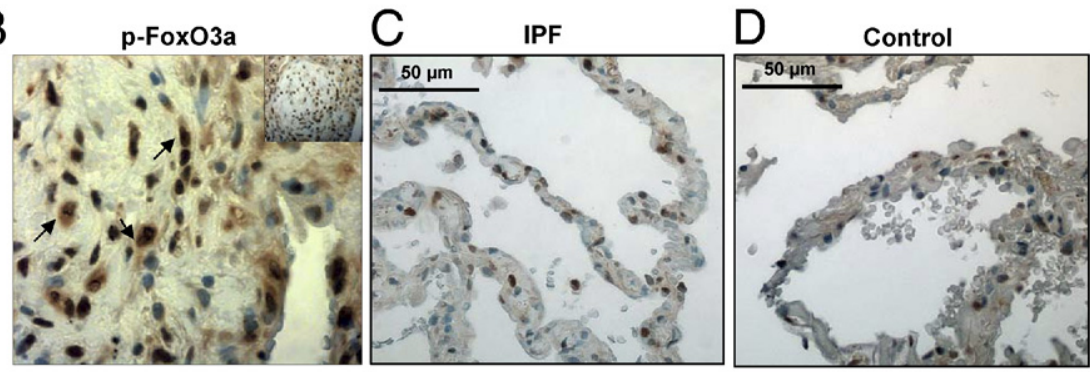

E IPF non-fibrotic region

F IPF fibroblastic foci

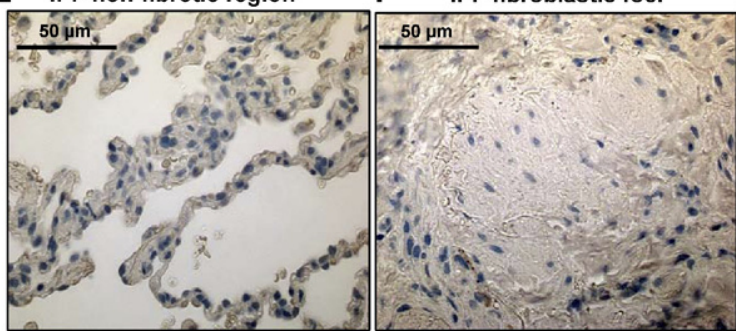

G

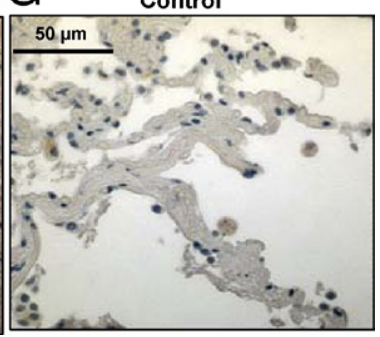

Figure 6. Cells within IPF fibroblastic foci are immunoreactive for inactive FoxO3a. IHC was performed on lung tissue from patients with IPF or patients with histologically normal lungs ( $n=$ 3 for both) using an anti-p-FoxO3a antibody. The specimens were counterstained with hematoxylin. A: Left and middle: Representative p-FoxO3a staining of a fibroblastic focus in IPF patient tissue is shown. Original magnification, $\times 40$. Many cells in the fibroblastic focus display strong immunoreactivity for p-FoxO3a (arrows). Right: Representative FoxO3a staining of a fibroblastic focus in IPF patient tissue is shown. Original magnification, $\times 40$. B: Increased magnification of a $\times 40$ image shown as an insert to illustrate nuclear and cytoplasmic staining of p-FoxO3a in the fibroblastic focus (arrows). C: Representative p-FoxO3a staining of the nonfibroblastic foci area of IPF patient tissue is shown. Original magnification, $\times 40$. The number of cells immunoreactive for p-FoxO3a is relatively low compared with that of cells in fibroblastic foci in A. D: A nonfibrotic lung control specimen stained for p-FoxO3a using 3,3-diaminobenzidine substrate. Original magnification, $\times 40$. E-G: Nonfibrotic region $(\mathbf{E})$ and fibroblastic foci (F) of IPF tissue and alveoli of control lung tissue (G) were stained with secondary antibody only (negative control). Original magnification, $\times 40$. proliferation increased approximately 30\% (Figure 5E). However, DA had a minimal effect on control fibroblast proliferation. More important, inhibition of high Akt activity by overexpression of DA suppressed IPF fibroblast proliferation by $29 \%$ (Figure 5E), whereas HA only marginally augmented IPF fibroblast proliferation. Taken together, these data indicate that inactivation of FoxO3a by inappropriately high Akt activity at least partially underlies the ability of IPF fibroblasts to circumvent the proliferationsuppressive effects of polymerized collagen.

\section{Myofibroblasts within IPF Fibroblast Foci Contain Inactive FoxO3a}

The fibroblastic focus is the sentinel morphological lesion of IPF. It is composed of a subepithelial accumulation of myofibroblasts in a type I collagen-rich matrix that expands and obliterates the alveolar airspace. ${ }^{24-27}$ We performed $\mathrm{IHC}$ to examine the expression of phosphorylated (inactive) FoxO3a within IPF fibroblastic foci and compared the results with human nonfibrotic control lung tissue. Consistent with our in vitro studies, cells with immunoreactivity to inactive $\mathrm{p}-\mathrm{FoxO} 3 \mathrm{a}$ were prevalent in fibroblastic foci (Figure 6A), whereas the overall expression of FoxO3a was low (Figure 6A). In accord with prior studies, our IHC analysis indicates that the cells within the fibroblastic foci stain prominently for $\alpha$-smooth muscle actin, identifying them as myofibroblasts (see Supplemental Figure S1B at http://ajp.amjpathol.org). Interest- ingly, unlike our in vitro studies indicating a predominantly cytoplasmic location of p-FoxO3a in IPF fibroblasts, the $\mathrm{IHC}$ staining pattern suggested both a cytoplasmic and a nuclear location for inactive FoxO3a in myofibroblasts within the fibroblastic focus (Figure 6B). Furthermore, normal-appearing alveoli adjacent to fibroblastic foci were largely devoid of p-FoxO3a-immunoreactive cells (Figure 6C). An analysis of human control lung tissue displayed a paucity of immunoreactive cells within anatomically normal-appearing alveolar walls (Figure 6D). Uninjured alveoli contain largely nonproliferating cells, and it is expected that FoxO3a would be active in these cells. No significant immunoreactivity was seen in either nonfibrotic regions or fibroblastic foci of IPF lung tissue or control specimens stained with secondary antibody only, respectively (Figure 6, E-G). Taken together, our $\mathrm{IHC}$ analysis strongly suggests that myofibroblasts within the fibroblastic focus display immunoreactivity for inactive FoxO3a and correlate with our in vitro analysis of IPF fibroblasts, which also show the presence of inactive FoxO3a in these cells.

\section{Discussion}

IPF is characterized by the relentless accumulation of IPF fibroblasts in fibroblastic foci, resulting in obliteration of alveoli with collagen-rich fibrotic tissue. Unlike normal fibroblasts, whose proliferation is suppressed by polymerized type I collagen, the IPF fibroblast phenotype is 
IPF fibroblasts

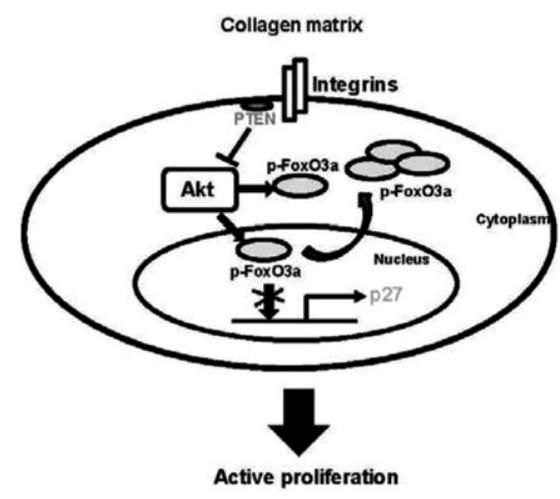

Control fibroblasts

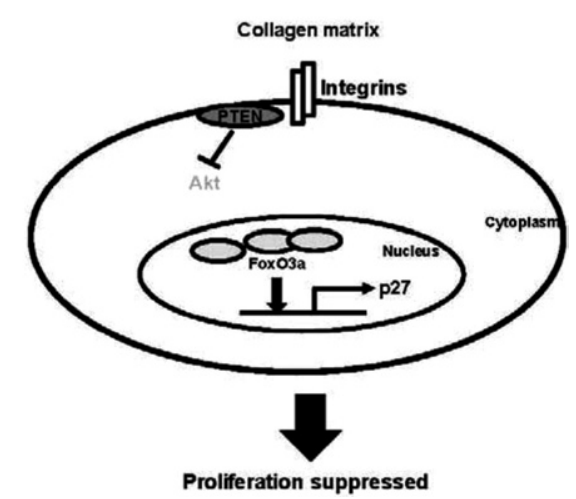

Figure 7. Schematic illustration of the IPF fibroblast pathological proliferation signaling pathway. When IPF fibroblasts interact with polymerized collagen, Akt activity is inappropriately high as a result of low PTEN activity. Akt phosphorylates and inactivates FoxO3a by promoting its translocation to the cytoplasm. Furthermore, p-FoxO3a in the nucleus cannot bind to DNA because of Ser253 phosphorylation. FoxO3a is then unable to activate its target gene $\mathrm{p} 27$, and proliferation is not suppressed (left). In contrast, when control fibroblasts interact with polymerized collagen, PTEN activity remains high, suppressing Akt activity. FoxO3a remains active and accumulates in the nucleus, promoting p27 expression and suppressing proliferation (right) characterized by an ability to escape the proliferationsuppressive properties of polymerized collagen. Although the precise molecular mechanisms underlying the ability of IPF fibroblasts to proliferate abnormally on collagen remain to be fully elucidated, we found that IPF fibroblasts display inappropriately low PTEN activity that aberrantly activates the PI3K-Akt pathway and promotes proliferation. $^{8}$ FoxO3a is a transcription factor that increases expression of the cell cycle inhibitor p27. When FoxO3a becomes active, it inhibits proliferation. In this study, we demonstrate that when IPF fibroblasts interact with polymerized collagen, FoxO3a is largely inactive, facilitating fibroblast proliferation. In contrast, when control nonfibrotic fibroblasts interact with polymerized collagen, FoxO3a is active, thereby suppressing proliferation. We demonstrate that, in IPF fibroblasts, FoxO3a is phosphorylated and inactivated by the abnormally high Akt activity resulting from inappropriately low PTEN function (Figure 7). Together, our results have identified a critical role for the PTEN-Akt-FoxO3a signal axis in conferring IPF fibroblasts with their pathological ability to proliferate in a collagen-rich proliferation-prohibitive environment.

The FoxO3a transcriptional factor inhibits cell cycle progression by promoting $\mathrm{G}_{1}$ arrest. It does so by upregulating the expression of $\mathrm{p} 27$, a negative regulator of $\mathrm{G}_{1}$ to $\mathrm{S}$ cell cycle transition. ${ }^{10}$ FoxO3a function is negatively regulated by the PI3K-Akt signal pathway. ${ }^{12}$ The activation of Akt phosphorylates and inactivates FoxO3a. Akt can phosphorylate three FoxO3a Ser-Thr residues (Thr32, Ser253, and Ser315), preferring Ser253. ${ }^{19,20}$ Aktdependent phosphorylation of FoxO3a facilitates translocation of FoxO3a from the nucleus to the cytoplasm, thus abrogating FoxO3a transcriptional activity. ${ }^{21-23}$ Crystal structure analysis also revealed that Ser253 phosphorylation of FoxO3a by Akt inhibits its DNA binding ability. ${ }^{28}$ In this study, we demonstrate that when human IPF fibroblasts are cultured on polymerized collagen, Ser253 of FoxO3a is highly phosphorylated and inactive. However, the level of $\mathrm{p}-\mathrm{FoxO} 3 \mathrm{a}$ is much lower in nonfibrotic control fibroblasts. By using inhibitors of Akt, we demonstrate that the inappropriately high Akt activity in IPF fibroblasts is responsible for the phosphorylation and inactivation of FoxO3a. In addition, we show that overexpression of PW in IPF fibroblasts reduces the level of $\mathrm{p}-\mathrm{FoxO} 3 \mathrm{a}$. Our studies strongly suggest that low PTEN activity in IPF fibroblasts aberrantly activates Akt, which, in turn, effectively phosphorylates and inactivates FoxO3a.

We found that the levels of $\mathrm{p}-\mathrm{FoxO} 3 \mathrm{a}$ are high in the cytoplasm of IPF fibroblasts and relatively low in the nucleus when the cells are cultured on collagen in vitro. However, DA clearly decreased the level of inactive FoxO3a in the cytoplasm. In contrast, the FoxO3a level significantly increased in the nucleus when DA was expressed, consistent with the idea that FoxO3a transcriptional activity is largely abrogated in IPF fibroblasts. This is also supported by our finding that p27 levels are low when IPF fibroblast are cultured on polymerized collagen compared with nonfibrotic control fibroblasts, whose p27 levels increase during culture on collagen. The sentinel morphological region of IPF is the fibroblastic focus. It consists of fibroblasts within a collagen-rich matrix. ${ }^{29,30}$ Consistent with our in vitro results showing that primary cultures of IPF fibroblasts contain abundant p-FoxO3a, when we analyzed human IPF lung tissue specimens, we found that cells within the fibroblastic focus were immunoreactive for $\mathrm{p}$-FoxO3a. Interestingly, p-FoxO3a was located within both the cytoplasm and the nucleus. This is somewhat at odds with our in vitro Western blot analysis data: we found that little p-FoxO3a could be detected within the nucleus of IPF fibroblasts when cultured on polymerized collagen. The explanation for this is not clear but may be related to differences in the microenvironment of the fibroblastic foci and the simplified in vitro collagen matrix assay. Another possibility is that there may be a difference in the sensitivity of the antibodies used for the detection of $\mathrm{p}-\mathrm{FoxO} 3 \mathrm{a}$ in fibroblasts cultured on collagen and in IPF patient specimens. Nevertheless, FoxO3a is found as a highly phosphorylated form in IPF fibroblasts cultured on collagen matrix or in cells within fibroblastic foci of IPF patient specimens. Prior studies ${ }^{31,32}$ documented that, when FoxO3a is phosphorylated, the DNA binding affinity is altered such that it has a reduced ability as a transcriptional activator to bind its substrate target. Therefore, our in vitro and in vivo studies demonstrate that FoxO3a activity is suppressed in IPF and strongly suggest that the alteration of FoxO3a activity can be responsible for the abnormal proliferative properties of IPF fibroblasts. 
Because FoxO3a regulates fibroblast proliferation, we attempted to determine whether there was a correlation between p-FoxO3a and $\mathrm{Ki}-67$ expression in IPF patient specimens. We found that relatively few cells expressed $\mathrm{Ki}-67$ in fibroblastic foci of IPF patient specimens, making it difficult to correlate $\mathrm{p}-\mathrm{FoxO} 3 \mathrm{a}$ levels with proliferation in vivo. A possible explanation for this is that proliferation is dynamic and occurs relatively rapidly. In the absence of longitudinal human tissue samples, a comprehensive analysis of $\mathrm{p}-\mathrm{FoxO} 3 \mathrm{a}$ expression and proliferation markers is not possible.

Furthermore, a direct comparison of FoxO3 activity in the in vitro assay with our in vivo analysis was also difficult. For example, we measured FoxO3a activity and p27 expression in IPF fibroblasts derived from patients with IPF on collagen at short time points. Unlike our in vitro assay, fibroblastic foci in IPF tissue likely develop over a longer period. Differences in FoxO3a activity between our in vitro and in vivo studies likely reflect these different biological conditions. Nevertheless, the predominant form of FoxO3a in IPF fibroblasts in vitro and in IPF fibroblastic foci in vivo is inactive $\mathrm{FoxO} 3$.

The role of FoxO3a in disease states is beginning to come into focus. ${ }^{33,34}$ The loss of FoxO3a promotes the growth of cancer cells. ${ }^{34}$ Regarding fibrosis, a recent study ${ }^{31}$ has linked inactivation of FoxO3a by PI3K-Akt to mesenchymal cell dysfunction and renal fibrosis in an animal model of diabetic kidney disease. ${ }^{35}$ Our studies provide evidence that in IPF, FoxO3a is largely inactive, thereby facilitating IPF fibroblast proliferation. In conclusion, our data suggest that the pathological IPF fibroblast phenotype is characterized by an aberrant PTEN-PI3K axis, resulting in inappropriately high Akt activity. This high Akt activity phosphorylates and inactivates $\mathrm{FoxO} 3 \mathrm{a}$ function, resulting in low p27 expression. Our data suggest that this confers IPF fibroblasts with the ability to circumvent the proliferation-suppressive effect of polymerized collagen.

\section{References}

1. Ryu JH, Colby TV, Hartman TE: Idiopathic pulmonary fibrosis: current concepts. Mayo Clin Proc 1998, 73:1085-1101

2. Gharaee-Kermani M, Gyetko MR, Hu B, Phan SH: Pharm: new insights into the pathogenesis and treatment of idiopathic pulmonary fibrosis: a potential role for stem cells in the lung parenchyma and implications for therapy. Pharm Res 2007, 24:819-841

3. Kuzuya M, Satake S, Ramos MA, Kanda S, Koike T, Yoshino K, Ikeda $S$, Iguchi A: Induction of apoptotic cell death in vascular endothelial cells cultured in three-dimensional collagen lattice. Exp Cell Res 1999, 248:498-508

4. Xia H, Nho RS, Kahm J, Kleidon J, Henke CA: Fibroblast survival in response to contraction of type I collagen matrices via a beta 1 integrin viability signaling pathway. J Biol Chem 2004, 279:3302433034

5. Nho RS, Xia H, Kahm J, Kleidon J, Diebold D, Henke CA: Role of integrin-linked kinase in regulating phosphorylation of Akt and fibroblast survival in type I collagen matrices through a beta1 integrin viability signaling pathway. J Biol Chem 2005, 280:26630-26639

6. Nho RS, Xia H, Diebold D, Kahm J, Kleidon J, White E, Henke CA: PTEN regulates fibroblast elimination during collagen matrix contraction. J Biol Chem 2006, 281:33291-33301

7. Xia H, Nho RS, Kahm J, Kleidon J, Henke CA: Focal adhesion kinase is upstream of phosphatidylinositol 3-kinase/Akt in regulating fibro- blast survival in response to contraction of type I collagen matrices via a beta 1 integrin viability signaling pathway. J Biol Chem 2004 , 279:33024-33034

8. Xia H, Diebold D, Nho R, Perlman D, Kleidon J, Kahm J, Avdulov S, Peterson M, Nerva J, Bitterman P, Henke C: Pathological integrin signaling enhances proliferation of primary lung fibroblasts from patients with idiopathic pulmonary fibrosis. J Exp Med 2008, 205 : 1659-1672

9. Xia H, Khalil W, Kahm J, Jessurun J, Kleidon J, Henke CA: Pathologic caveolin-1 regulation of PTEN in idiopathic pulmonary fibrosis. Am J Pathol 2010, 176:2626-2637

10. Nakao T, Geddis AE, Fox NE, Kaushansky K: PI3K/Akt/FOXO3a pathway contributes to thrombopoietin-induced proliferation of primary megakaryocytes in vitro and in vivo via modulation of p27(Kip1). Cell Cycle 2008, 7:257-266

11. Zhao Y, Fei M, Wang Y, Lu M, Cheng C, Shen A: Expression of Foxo3a in non-Hodgkin's lymphomas is correlated with cell cycle inhibitor p27. Eur J Haematol 2008, 81:83-93

12. Li CJ, Chang JK, Chou CH, Wang GJ, Ho ML: The PI3K/Akt/FOXO3a/ p27Kip1 signaling contributes to anti-inflammatory drug-suppressed proliferation of human osteoblasts. Biochem Pharmacol 2010, 79: 926-937

13. Castrillon DH, Miao L, Kollipara R, Horner JW, DePinho RA: Suppression of ovarian follicle activation in mice by the transcription factor Foxo3a. Science 2003, 301:215-218

14. Mikse OR, Blake DC Jr, Jones NR, Sun YW, Amin S, Gallagher CJ, Lazarus P, Weisz J, Herzog CR: FOXO3 encodes a carcinogenactivated transcription factor frequently deleted in early-stage lung adenocarcinoma. Cancer Res 2010, 70:6205-6215

15. Sunters A, Fernández de Mattos S, Stahl M, Brosens JJ, Zoumpoulidou G, Saunders CA, Coffer PJ, Medema RH, Coombes RC, Lam EW: FoxO3a transcriptional regulation of Bim controls apoptosis in paclitaxel-treated breast cancer cell lines. J Biol Chem 2003 278:49795-49805

16. Chapuis N, Park S, Leotoing L, Tamburini J, Verdier F, Bardet V, Green AS, Willems L, Agou F, Ifrah N, Dreyfus F, Bismuth G, Baud V, Lacombe C, Mayeux P, Bouscary D: $I_{\kappa}$ B kinase overcomes PI3K/Akt and ERK/MAPK to control FOXO3a activity in acute myeloid leukemia. Blood 2010, 116:4240-4250

17. Brunet A, Park J, Tran H, Hu LS, Hemmings BA, Greenberg ME: Protein kinase SGK mediates survival signals by phosphorylating the forkhead transcription factor FKHRL1 (FOXO3a). Mol Cell Biol 2001, 21:952-965

18. Nho RS, Kahm J: $\beta 1$-Integrin-collagen interaction suppresses FoxO3a by the coordination of Akt and PP2A. J Biol Chem 2010, 285:14195-14209

19. Zheng WH, Kar S, Quirion R: Insulin-like growth factor-1-induced phosphorylation of the forkhead family transcription factor FKHRL1 is mediated by Akt kinase in PC12 cells. J Biol Chem 2000, 275:3915239158

20. Yusuf L, Zhu X, Kharas MG, Chen J, Fruman DA: Optimal B-cell proliferation requires phosphoinositide 3-kinase-dependent inactivation of FOXO transcription factors. Blood 2004, 104:784-787

21. Chen YR, Liu MT, Chang YT, Wu CC, Hu CY, Chen JY: Epstein-Barr virus latent membrane protein 1 represses DNA repair through the PI3K/Akt/FOXO3a pathway in human epithelial cells. J Virol 2008 82:8124-8137

22. Nakae J, Barr V, Accili D: Differential regulation of gene expression by insulin and IGF-1 receptors correlates with phosphorylation of a single amino acid residue in the forkhead transcription factor FKHR. EMBO J 2000, 19:989-996

23. Tang ED, Nuñez G, Barr FG, Guan KL: Negative regulation of the forkhead transcription factor FKHR by Akt. J Biol Chem 1999, 274 16741-16746

24. Sanders YY, Tollefsbol TO, Varisco BM, Hagood JS: Epigenetic regulation of thy- 1 by histone deacetylase inhibitor in rat lung fibroblasts. Am J Respir Cell Mol Biol 2011, 45:16-23

25. Leppäranta O, Pulkkinen V, Koli K, Vähätalo R, Salmenkivi K, Kinnula VL, Heikinheimo M, Myllärniemi M: Transcription factor GATA-6 is expressed in quiescent myofibroblasts in idiopathic pulmonary fibrosis. Am J Respir Cell Mol Biol 2010, 42:626-632

26. Sanders YY, Pardo A, Selman M, Nuovo GJ, Tollefsbol TO, Siegal GP Hagood JS: Thy-1 promoter hypermethylation: a novel epigenetic patho- 
genic mechanism in pulmonary fibrosis. Am J Respir Cell Mol Biol 2008 , $39: 610-618$

27. White ES, Atrasz RG, Hu B, Phan SH, Stambolic V, Mak TW, Hogaboam CM, Flaherty KR, Martinez FJ, Kontos CD, Toews GB: Negative regulation of myofibroblast differentiation by PTEN (Phosphatase and Tensin Homolog Deleted on chromosome 10). Am J Respir Crit Care Med 2006, 173:112-121

28. Tsai KL, Sun YJ, Huang CY, Yang JY, Hung MC, Hsiao CD: Crystal structure of the human FoxO3a-DBD/DNA complex suggests the effects of post-translational modification. Nucleic Acids Res 2007, 35:6984-6994

29. Noble PW: Idiopathic pulmonary fibrosis: natural history and prognosis. Clin Chest Med 2006, 27:S11-S16

30. Crestani B, Marchand-Adam S, Fabre A, Dehoux M, Soler P: [Mechanisms in pulmonary fibrosis]. Rev Prat 2007, 57:2222-2226

31. Brunet A, Bonni A, Zigmond MZ, Lin MZ, Juo P, Hu LS, Anderson MJ, Arden KC, Blenis J, Greenberg ME: Akt promotes cell survival by phosphorylating and inhibiting a Forkhead transcription factor. Cell 1999, 96:857-868

32. Biggs WH 3rd, Meisenhelder J, Hunter T, Cavenee WK, Arden KC: Protein kinase B/Akt-mediated phosphorylation promotes nuclear exclusion of the winged helix transcription factor FKHR1. Proc Natl Acad Sci U S A 1999, 96:7421-7426

33. Ikeda J, Mamat S, Tian T, Wang Y, Rahadiani N, Aozasa K, Morii E: Tumorigenic potential of mononucleated small cells of Hodgkin lymphoma cell lines. Am J Pathol 2010, 177:3081-3088

34. Shukla S, Shukla M, Maclennan GT, Fu P, Gupta S: Deregulation of FOXO3A during prostate cancer progression. Int J Oncol 2009, 34: 1613-1620

35. Kato M, Yuan H, Xu ZG, Lanting L, Li SL, Wang M, Hu MC, Reddy MA, Natarajan R: Role of the Akt/FoxO3a pathway in TGF-beta1-mediated mesangial cell dysfunction: a novel mechanism related to diabetic kidney disease. J Am Soc Nephrol 2006, 17:3325-3335 